\title{
Cellular expression, localization and interactions of the product of the human MOST-1 gene associated with breast and prostate cancers
}

\author{
JEANNE M.M. TAN and VINCENT T.K. CHOW \\ Human Genome Laboratory, Department of Microbiology, Yong Loo Lin School of Medicine, \\ National University of Singapore, Kent Ridge, Singapore
}

Received July 31, 2006; Accepted September 18, 2006

\begin{abstract}
We previously isolated and characterized the novel human gene MOST-1 (C8orf17) that is ubiquitously expressed in all cancer cell lines tested but differentially expressed in normal adult tissues. MOST-1 maps to chromosome region $8 \mathrm{q} 24.2$ whose amplification is frequently associated with breast and prostate cancers. RT-PCR analyses of breast and prostatic biopsies revealed MOST-1 overexpression and/or amplification in high-grade carcinomas. We raised and characterized a polyclonal antibody against a MOST-1specific synthetic peptide. In vitro expression of MOST-1 protein revealed a tendency to exist as high molecular mass isoforms which are SDS-insoluble upon thermal stress. MOST-1 displayed cytoplasmic localization in four human cell lines (hTERT-HME1 normal mammary epithelial, MCF7 breast adenocarcinoma, PrEC normal prostate epithelial and DU145 prostate carcinoma), with polar expression during cell division. Knockdown of MOST-1 expression in DU145 cells resulted in reduced cell proliferation but enhanced apoptosis implying a putative mitogenic role of MOST-1. Yeast two-hybrid analyses demonstrated interaction with seven human proteins, most of which are overexpressed in tumors or involved in metabolic pathways. The interacting proteins were creatine kinase, Gardner feline sarcoma v-FGR oncogene product, telethonin, SNC73 protein, ferritin light chain, peripheral benzodiazepine receptor, and immunoglobulin $\mathrm{C}(\mu)$ and $\mathrm{C}(\delta)$ heavy chain. Co-immunoprecipitation assays validated the interactions of MOST-1 with the latter three proteins. Our results suggest that MOST-1 is associated with cell survival, proliferation and progression of cancer cells.
\end{abstract}

Correspondence to: Dr Vincent T. Chow, Human Genome Laboratory, Department of Microbiology, Yong Loo Lin School of Medicine, National University of Singapore, 5 Science Drive 2, Kent Ridge 117597, Singapore

E-mail: micctk@nus.edu.sg

Key words: MOST-1, c8orf17, aggregation, expression, localization, protein-protein interactions, breast cancer, prostate cancer

\section{Introduction}

We previously reported the cDNA sequence of a novel human gene isolated from the MOLT-4 T-lymphoblastic leukemia cell line (1). Designated MOST-1 and given the symbol C8orf17 by the Human Genome Organization (HUGO) Nomenclature Committee, this gene is ubiquitously expressed in all cancer cell lines tested, but differentially expressed in normal adult tissues. MOST-1 maps to chromosome 8q24.2, a region that is amplified in many breast and prostate cancers, and also the candidate site of potential oncogene(s) other than c-myc located at 8q24.1 (2,3). Genes amplified or overexpressed in high-grade cancers often play key roles in cell cycle regulation, and their amplification results in a loss of growth control. Elevated MOST-1 expression is observed in high-grade breast cancers, while MOST-1 shows increasing amplification with increasing grade of prostate carcinomas and is amplified 10-fold in high-grade tumors (1).

Being a multi-factorial disease, cancer requires multiple genetic aberrations to occur for its progression. The process of cancer progression and metastasis requires a large consortium of genes including oncogenes, motility factors and matrix metalloproteinases $(4,5)$. During this process, mutation and/or accumulation of excess cancer-related proteins may occur, culminating in a dysfunctional cell. Given that the MOST-1 gene is overexpressed or amplified in high-grade breast and prostate cancers, we hypothesized that there may be a role for MOST-1 in cancer progression. To gain insight into the putative functions of the MOST-1 protein in cancer, we characterized a polyclonal antibody raised against a MOST-1 synthetic peptide, and utilized it to determine the cellular localization of MOST-1. RNA interference (RNAi) and cell synchronization experiments were performed to investigate the involvement of MOST-1 in the cell cycle. We also report the results of yeast two-hybrid screening of a human bone marrow cDNA library and co-immunoprecipitation analyses for identifying interacting partner proteins of MOST-1.

\section{Materials and methods}

Computational analysis of the amino acid sequence of MOST-1. The amino acid sequence of MOST-1 was analyzed using various software programs (e.g. ProtParam) available from 
these websites, i.e. www.expasy.ch and http://bioportal.bic. nus.edu.sg/.

Generation of polyclonal antibody against MOST-1. Rabbit anti-MOST-1 polyclonal antibody was raised by immunization with a synthetic peptide Ac-TGPEQSDICHTGSEAR-NH (corresponding to amino acids 19-34 within the predicted hydrophilic and antigenic regions of MOST-1) conjugated to diphtheria toxoid (Mimotopes, Clayton, Australia). Prior to use, the GenBank database was screened using BLASTP to exclude homology of this peptide sequence to any known protein. Polyclonal antisera were raised according to previously described procedures $(6,7)$. Batchwise affinity purification was carried out according to the manufacturer's recommendation. Briefly, specific peptide covalently coupled to a thiopropylsepharose $6 \mathrm{~B}$ gel syringe column was washed 5 times with $8 \mathrm{ml}$ of PBS (pH 7.4). Serum $(5 \mathrm{ml})$ followed by PBS $(3 \mathrm{ml})$ was drawn into the syringe before sealing the nozzle. The syringe contents were mixed at $4^{\circ} \mathrm{C}$ overnight. The solution was then expelled from the syringe, washed 5 times with PBS followed by 3 washes with saline. Seven $\mathrm{ml}$ of $100 \mathrm{mM}$ glycine/ $\mathrm{HCl}$ buffer $(\mathrm{pH} 2.5$ ) was mixed with gel slurry for $1 \mathrm{~min}$ before expulsion from the syringe. Elution was repeated 2 more times followed by 2 washes with saline. The procedure was repeated with $100 \mathrm{mM}$ glycine/ $\mathrm{NaOH}$ buffer $(\mathrm{pH} 11.5)$. All solutions were pooled, and $\mathrm{pH}$ of the antibody solution was adjusted to 7.0 using $5 \%$ phosphoric acid. The antibody was then concentrated in $5 \mathrm{ml}$ using an Amicon ultrafiltration apparatus model M3. Antibody was then aliquoted and stored at $-20^{\circ} \mathrm{C}$.

In vitro transcription and translation (TNT) experiments to verify the specificity of the polyclonal antibody. The MOST-1 gene was cloned into a pBluescript II SK vector (Stratagene, La Jolla, CA) for expression in the T7 Quick Coupled TNT system (Promega, Madison, WI). In vitro TNT experiments were performed with or without Redivue L-[S $\left.\mathrm{S}^{35}\right]$-labeled methionine (Amersham Biosciences, Piscataway, NJ). Briefly, TNT mix with $1 \mu \mathrm{g}$ of plasmid containing the MOST-1 insert and $1 \mathrm{mM}$ labeled or unlabeled methionine were incubated at $30^{\circ} \mathrm{C}$ for $90 \mathrm{~min}$. The translated products $(5 \mu \mathrm{l})$ were then electrophoresed in an SDS-10\% polyacrylamide gel. Western blot detection as described below was performed on the unlabeled reaction mix lane, while the labeled reaction mix lane was exposed to X-ray film overnight. TNT experiments with unlabeled methionine were carried out as above, and TNT products were differentially subjected to either $95^{\circ} \mathrm{C}$ for 5 min or incubation with $2 \%$ SDS or both treatments. Products or untreated rabbit lysate only $(5 \mu 1)$ were then electrophoresed in an SDS-10\% polyacrylamide gel before Western blot analysis as described below. Experiments were duplicated.

Cell culture. MOLT-4 T-lymphoblastic leukemia and baby hamster kidney (BHK) cell lines were maintained with RPMI-1640 medium supplemented with $10 \%$ fetal bovine serum (FBS). Cells were passaged every 3 days at ratios of 1:4 and 1:6, respectively. The hTERT-HME1 mammary epithelial cell line was maintained in MCDB 170 medium supplemented with $52 \mu \mathrm{g} / \mathrm{ml} \mathrm{BPE,} 0.5 \mu \mathrm{g} / \mathrm{ml}$ hydrocortisone,
$10 \mathrm{ng} / \mathrm{ml} \mathrm{hEGF}, 5 \mu \mathrm{g} / \mathrm{ml}$ insulin, $50 \mu \mathrm{g} / \mathrm{ml}$ gentamicin and $50 \mathrm{ng} / \mathrm{ml}$ amphotericin B. PrEC prostate epithelial cells were maintained in PrEGM basal medium (Clonetics, Baltimore, $\mathrm{MD})$. Both epithelial cell lines were passaged every 7 days at a ratio of 1:3. MCF7 breast adenocarcinoma cells were cultured in DMEM supplemented with 10\% FBS. DU145 prostate carcinoma cells were maintained in MEM supplemented with $10 \%$ FBS. Cancer cell lines were passaged every 3 days at a ratio of $1: 4$.

Total protein extraction and subcellular fractionation. Total protein extracts were prepared by culturing cells to $90 \%$ confluence before washing thrice with cold PBS ( $\mathrm{pH} 7.4$ ). Lysis buffer I (1\% sodium deoxycholate, $1 \%$ Triton X-100, $100 \mathrm{mM}$ Tris- $\mathrm{HCl} \mathrm{pH} 7.5$ and $150 \mathrm{mM} \mathrm{NaCl}$ ) with complete protease inhibitor cocktail (Roche, Alameda, CA) was added, and incubated on ice for $30 \mathrm{~min}$ with occasional mixing before centrifugation at $10,000 \times \mathrm{g}$ at $4^{\circ} \mathrm{C}$ for $15 \mathrm{~min}$. The supernatant was then harvested. Subcellular fractions of cultured cells were prepared as previously described with modifications $(6,7)$. Briefly, cells were cultured to $90 \%$ confluence before washing thrice with cold PBS. Lysis buffer II $(0.02 \%$ Nonidet-P40, $150 \mathrm{mM} \mathrm{NaCl}, 2 \mathrm{mM} \mathrm{MgCl} 2,3 \mathrm{mM}$ $\mathrm{CaCl}_{2}$ and $3 \mathrm{mM} \mathrm{DTT}$ ) with complete protease inhibitor cocktail was added, and incubated on ice for $15 \mathrm{~min}$ with occasional mixing before centrifugation at $500 \mathrm{x} \mathrm{g}$ at $4^{\circ} \mathrm{C}$ for $5 \mathrm{~min}$. The pellet representing the nuclear fraction was processed with lysis buffer I. The supernatant was harvested and centrifuged at $100,000 \mathrm{x} \mathrm{g}$ at $4^{\circ} \mathrm{C}$ for $1 \mathrm{~h}$. The resulting pellet representing the microsomal fraction was processed in a similar way as the nuclear pellet. The final supernatant containing the cytosol fraction was brought to a final concentration of $1 \%$ sodium deoxycholate and $1 \%$ Triton X-100.

Western blot analysis. Protein lysate (10 $\mu \mathrm{g}$ per lane) was subjected to $12 \%$ polyacrylamide-SDS gel electrophoresis in Laemmli running buffer, and electroblotted onto polyvinylidene difluoride (PVDF) membrane in CAPS transfer buffer $(10 \mathrm{mM}$ CAPS, $10 \%$ methanol). Membranes were blocked in $3 \%$ bovine serum albumin, $0.25 \%$ gelatin, $150 \mathrm{mM} \mathrm{NaCl}$ and $15 \mathrm{mM}$ Tris- $\mathrm{HCl}(\mathrm{pH} 7.4)$ at room temperature for $1 \mathrm{~h}$, and washed twice with TBST buffer $(150 \mathrm{mM} \mathrm{NaCl}, 15 \mathrm{mM}$ Tris- $\mathrm{HCl}$ pH 7.4 and $0.05 \%$ Tween-20). Each blot was incubated with affinity-purified polyclonal antibody (diluted 1:1,000 with TBST) at $4^{\circ} \mathrm{C}$ overnight, washed twice with TBST, followed by incubation with biotinylated goat anti-rabbit $\operatorname{IgG}$ (diluted 1:3,000 with TBST) for $1 \mathrm{~h}$ at room temperature, washed twice with TBST, and reacted with streptavidin conjugated with alkaline phosphatase (diluted 1:10,000 with alkaline phosphatase buffer containing $100 \mathrm{mM} \mathrm{NaCl}, 15 \mathrm{mM}$ Tris$\mathrm{HCl} \mathrm{pH} 7.4$ and $0.05 \%$ Tween-20). Immunoreactive bands were visualized by color development with NBT and BCIP substrates. Benchmark protein ladder markers (Invitrogen, Carlsbad, CA) were concurrently electrophoresed, transferred, cut, and stained with Coomassie blue. Molecular masses of immunoreactive bands were then estimated from log graph plots. Experiments were performed in triplicate.

Indirect immunofluorescence. Cells were cultured on glass coverslips in 24-well plates until 70-80\% confluence, washed 
thrice with cold PBS, fixed with $3 \%$ paraformaldehyde at room temperature for $10 \mathrm{~min}$, washed with PBS, permeabilized with $0.1 \%$ Triton $\mathrm{X}-100$ for $1 \mathrm{~min}$, washed twice with PBS before quenching aldehyde groups with $5 \mathrm{mM} \mathrm{NH}_{4} \mathrm{Cl}$ in PBS. Cells were then blocked with $0.1 \%$ FBS in PBS at room temperature for $30 \mathrm{~min}$ before incubation with polyclonal antibody (diluted 1:100 with blocking solution) overnight at $4^{\circ} \mathrm{C}$ in a humidified chamber. Cells were then washed with three changes of blocking solution over $30 \mathrm{~min}$ before incubation with FITC-conjugated goat anti-rabbit IgG antibody (diluted 1:10 with blocking solution) at room temperature for $1 \mathrm{~h}$. This was followed by 3 washes with blocking solution and one wash with PBS before mounting coverslips on ethanolcleaned slides, and viewed using a Zeiss LSM 510 laser scanning confocal inverted microscope and a Zeiss fluorescence light microscope. Experiments were performed in triplicate.

Cell synchronization studies. Cell synchronization was performed as previously described with modifications (8) on MCF7 breast carcinoma cells and hTERT-HME1 normal mammary epithelial cells to compare MOST-1 expression in cancer and normal cells. Briefly, cells were grown to $50 \%$ confluence, medium containing $0.5 \mathrm{mM}$ mimosine (Sigma, St. Louis, MO) was added, and incubated at $37^{\circ} \mathrm{C}$ for $24 \mathrm{~h}$. Medium was then removed, cells were washed with PBS, and normal growth medium was added. Cells were then harvested at $0,2,4,6,8,10,12,36,48 \mathrm{~h}$, and subjected to immunofluorescence confocal microscopy and flow cytometry. Experiments were duplicated.

MOST-1 expression studies. MOST-1 was amplified using primers pcDNAF (5'-CACCATGGGAGAATGTCCCCACC3') and pcDNARev (5'-CCATGTTGTCCAGGCTGGTC TCG-3') to generate a product incorporating the MOST-1 ORF of $297 \mathrm{bp}$, and cloned into a pcDNA3.1 expression vector (Invitrogen). Clones were sequenced to ensure that the protein was in-frame, and expression was checked via in vitro translation as recommended by the manufacturer (Promega). Cells were then transfected with Lipofectamine 2000 (Invitrogen) according to recommendations. Extraction of cell lysate at different time-points followed by Western blot analysis using anti-V5-AP (Invitrogen) was performed to determine the optimal time for expression. At the optimal time of $36 \mathrm{~h}$, cells were harvested for RT-PCR analysis using MOST-1 gene-specific primers TR4.2 and TF2 as well as G3PDH housekeeping primers (1).

Knockdown of MOST-1 expression by small interfering RNA. Duplex RNA (Dharmacon, Chicago, IL) targeting the ORF start site was constructed with the sequences 5'-UGUCCCCA CCUCGUGGAUGdTdT-3' and 5'-CAUCCACGAGGUGGG GACAdTdT-3'. The oligonucleotides were annealed according to the manufacturer's protocol, with $3 \mu \mathrm{l}$ of $20 \mathrm{mM}$ duplex RNA used per well. As a control for specificity, we used a scrambled oligoribonucleotide pair with the sequences 5'GCGCGCUUUGUAGGAUUCGdTdT-3' and 5'-CGAAUCC UACAAAGCGCGCdTdT-3'. To test the cell lines for RNAi silencing, we employed luciferase GL3-duplex with the sequences 5'-CUUAGCCUGAGUACUUCGAdTdT-3' and 5'-UCGAAGUACUCAGCGUAAGdTdT-3', and transfected the cells together with pGL3-control vector (Promega). Cells were transfected with Lipofectamine 2000 according to the recommended protocol, cells were harvested after $36 \mathrm{~h}$, and RNA was extracted using the Rneasy kit (Qiagen, Hilden, Germany) for RT-PCR analysis. Cells were also subjected to the cell proliferation assay via BrdU labeling and to the TUNEL assay. For the cell proliferation assay, the thymidine analog, 5-bromo-2-deoxyuridine (BrdU) was added for the last $6 \mathrm{~h}$ of culture. Cells $\left(10^{6}\right.$ per $\left.\mathrm{ml}\right)$ were then fixed in $70 \%$ ethanol for $4 \mathrm{~h}$ prior to acid treatment for $15 \mathrm{~min}$. Cells were then labeled with anti-BrdU-FITC, and propidium iodide (PI) was added as a counter stain before flow cytometric analysis by the Coulter imaging system. In the TUNEL assay (Clontech, Palo Alto, CA), cells $\left(10^{6}\right.$ per $\left.\mathrm{ml}\right)$ were fixed in $4 \%$ paraformaldehyde for $20 \mathrm{~min}$ prior to permeabilization with $0.1 \%$ Triton X-100 for $5 \mathrm{~min}$ on ice. Cells were then labeled with terminal deoxynucleotidyl transferase (TdT)-mediated dUTP nick-end labeling with FITC-dUTP. Prior to flow cytometric analysis, PI was added as a counter stain. Experiments were duplicated.

Yeast two-hybrid interaction screening. Yeast two-hybrid screening was carried out using the Matchmaker GAL4 TwoHybrid system (Clontech) as previously described with modifications (8). Briefly, the entire ORF of MOST-1 was generated using high-fidelity Pfu DNA polymerase (Promega). The amplicon and pAS2-1 vector were then digested with the relevant restriction enzymes, ligated overnight at $4^{\circ} \mathrm{C}$, and transformed into competent $E$. coli DH5 $\alpha$ cells. In-frame cloning of the bait construct was confirmed by sequencing prior to transformation into PJ69-2A yeast cells using the lithium acetate-based method. Fusion proteins from yeast lysates were then checked with MOST-1-specific antibody by Western blotting. A Matchmaker cDNA library derived from human bone marrow was screened via yeast mating by incubating $1 \mathrm{ml}$ of the library culture with the PJ69-2A bait culture in YPDA/kan overnight at $30^{\circ} \mathrm{C}$ with shaking at $30 \mathrm{rpm}$. Mating cultures were checked under phase-contrast microscopy for the presence of zygotes after $20 \mathrm{~h}$, and allowed to mate for a further $4 \mathrm{~h}$ after which they were collected by centrifugation at $1,000 \mathrm{x} \mathrm{g}$ for $10 \mathrm{~min}$. The mating flask was rinsed twice with YPDA/kan, and the first pellet was resuspended before collecting cells by centrifugation. The cell pellet was then resuspended in $10 \mathrm{ml}$ of YPDA/kan. Of each 10-fold dilution $(1: 10,000,1: 1,000,1: 100$ and $1: 10)$ of the mating mixture $100 \mu 1$ was plated on SD/-Leu, SD/-Trp and SD/-Leu/-Trp plates to assay for mating efficiency, while the remaining mating mixtures (200 $\mu 1$ each) were spread onto SD/-Ade/His/-Leu/-Trp (QDO) plates. The plates were then incubated at $30^{\circ} \mathrm{C}$ for 21 days, colony appearance was checked every 3 days, and colonies were subcultured on fresh QDO plates. $\beta$-galactosidase colony lift filter assay, and direct colony PCR screening of positive clones to detect the presence of both bait and library were performed using FAD and RADO primers (8) and as well as with MOST-1-specific primers. Clones were sequenced and analyzed using BLASTN and BLASTP algorithms.

Co-immunoprecipitation experiments. The bait construct used in yeast two-hybrid experiments was digested with SalI and 


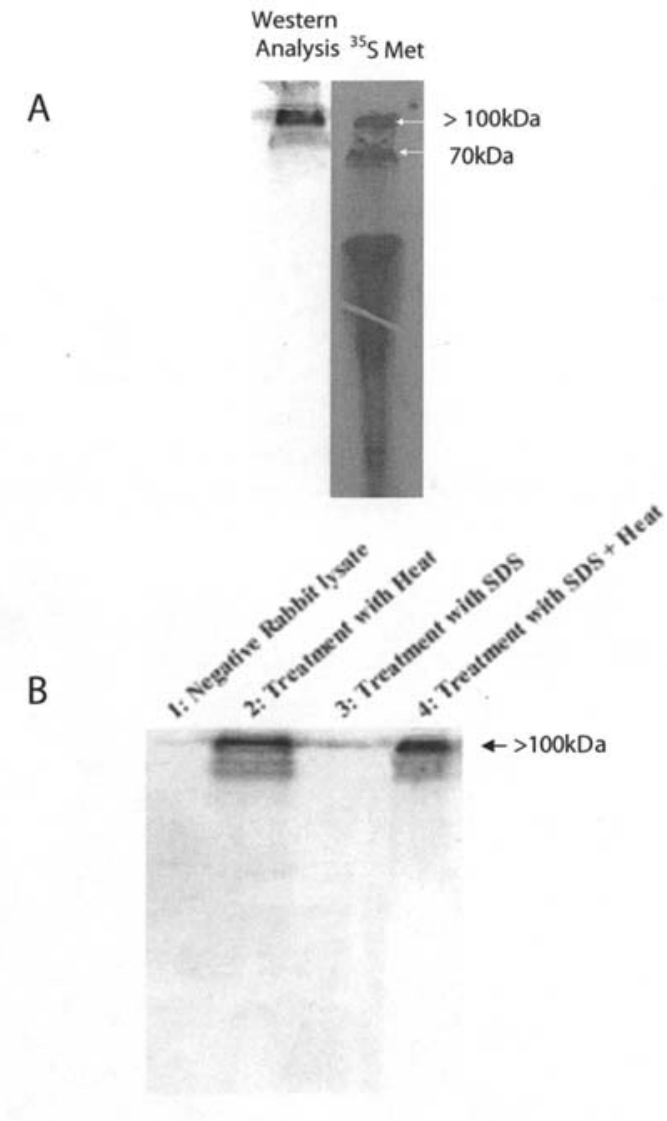

XhoI restriction enzymes and cloned into the pCMV-HA vector (Clontech) to generate pCMV-HA-MOST-1, while selected interactors were cloned into the pCMV-Myc vector (Clontech) using restriction enzymes EcoRI and XhoI. In-frame cloning was confirmed via sequencing. Plasmid DNA $(1 \mu \mathrm{g}$ each) was used for separate transfections with Cytofectene (Bio-Rad, Hercules, CA) according to the manufacturer's instructions. BHK cells grown to $\sim 80 \%$ confluence in RPMI-1640 supplemented with 10\% FBS and OPTI-MEM 1 (Gibco, Carlsbad, CA) were transfected, and incubated in $\mathrm{CO}_{2}$ for 24-48 h. Cells were washed thrice with cold PBS prior to incubation on ice for $30 \mathrm{~min}$ with mild lysis buffer

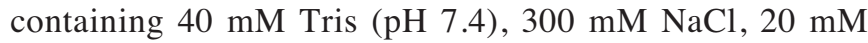
EDTA (pH 8), 20\% glycerol, 2\% Nonidet-P40 and complete protease inhibitor cocktail (Roche). Cell debris was removed by centrifugation at $10,000 \mathrm{x}$ g at $4^{\circ} \mathrm{C}$ for $15 \mathrm{~min}$. Lysates were then mixed in vitro, expressed proteins were coimmunoprecipitated overnight at $4^{\circ} \mathrm{C}$ with the respective antibodies, and subsequently pulled down with protein $\mathrm{A} / \mathrm{G}$ Plus agarose (Oncogene Science, Cambridge, MA) for $2 \mathrm{~h}$ before washing twice with PBS. SDS loading buffer was then added, the mixture heated at $95^{\circ} \mathrm{C}$ for 5 min before loading onto an SDS-PAGE gel, and subjected to Western blot analysis using the corresponding antibodies to the HA and Myc tags (8). Experiments were performed in triplicate.

\section{Results}

MOST-1 is predicted to be a highly insoluble protein. Computational analysis of the putative MOST-1 protein

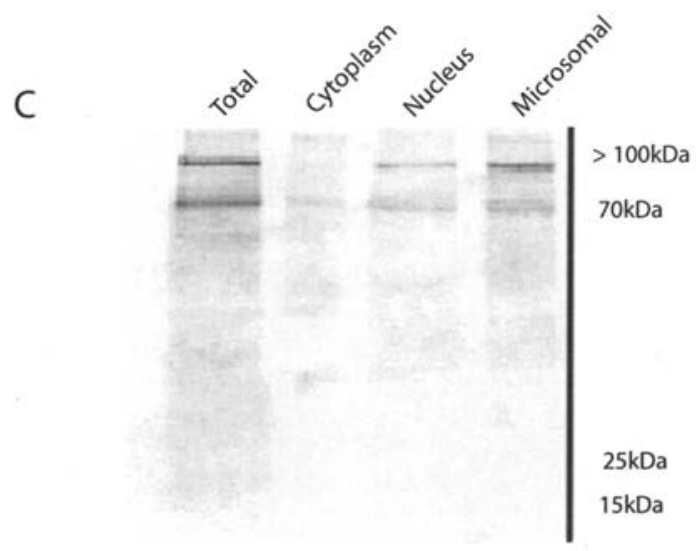

Figure 1. (A) Specific polyclonal antibody detection of high molecular mass MOST-1 protein expressed in transcription and translation (TNT) experiments. Lane 1, Western blot analysis of MOST-1 TNT product detected by affinity-purified polyclonal antibody showing specific recognition of MOST-1 protein of higher molecular mass. Lane 2, autoradiograph of $\mathrm{S}^{35}$-labeled MOST-1 TNT product showing multiple forms of synthesized MOST-1. (B) Differential treatment of aggregated MOST-1. Lane 1, Western blot analysis of negative control TNT rabbit reticulocyte lysate. Lane 2, Western blot analysis following heat treatment of in vitro translated MOST-1. Lane 3, Western blot analysis following SDS treatment of in vitro translated MOST-1. Lane 4, Western blot analysis following treatment of in vitro translated MOST-1 with heat and SDS. (C) Subcellular localization studies of MOST-1 by Western blot analysis of MOLT-4 cell lysate fractions. Lanes 1-4, total cell lysate, cytoplasmic, nuclear and microsomal fractions, respectively. The two major MOST-1 protein bands of $>100$ and $\sim 70 \mathrm{kDa}$ were present mainly in the microsomal and nuclear fractions.

revealed an $\mathrm{N}$-myristoylation site with glycine at position 2; casein kinase II phosphorylation sites with threonine at positions 19 and 29; protein kinase C phosphorylation sites with serine at positions 69 and 91. Serine at position 91 is predicted to be a site for O-glycosylation, while lysine 80 is a potential sumoylation site. The Protein Predict program predicted MOST-1 to be non-globular in nature, congruent with the predicted secondary structure with a predominant extended sheet structure spanning amino acids 7-11, 34-56, 60-64, 67-71 and 80-81. In addition, MOST-1 was predicted to be an unstable hydrophilic protein of $\sim 11 \mathrm{kDa}$ with a charge of 4.5 and an isoelectric point of 8.5. These analyses suggest that MOST-1 may be poorly soluble in nature, associate either with membranes or vesicular structures, and have a high propensity to aggregate.

The specific polyclonal antibody recognizes the high molecular mass form of MOST-1. The synthetic MOST-1 peptide TGPEQSDICHTGSEAR was selected on the basis of its high antigenicity and hydrophilicity as predicted by the GCG software PlotStructure. We generated three sets of polyclonal antisera against this synthetic peptide. Immuno-dot blot screening of these antisera showed hyperimmune antibodies reacting strongly to conjugated and unconjugated peptides, whereas their corresponding preimmune control sera were nonreactive (data not shown). Using a peptide-bound column, the strongest reacting polyclonal antibody (no. 235) was affinity-purified for use as a probe in Western blotting and confocal immunofluorescence analyses. Characterization of the affinity-purified polyclonal antibody revealed that it recognized 


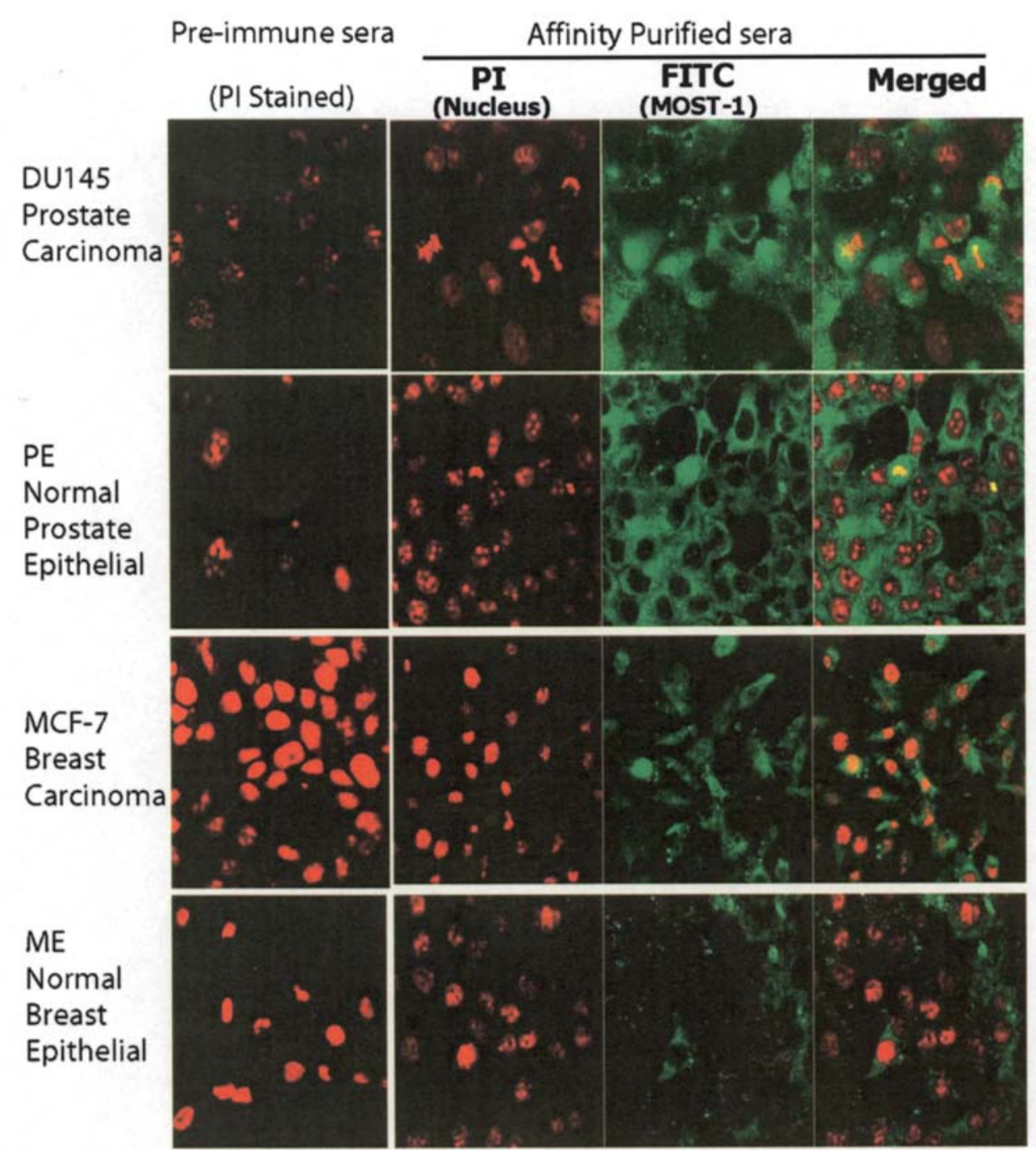

Figure 2. Comparison of subcellular distribution of MOST-1 protein in malignant and normal prostate and mammary cells by confocal microscopy. Column 1, cells incubated with pre-immune sera followed by FITC-labeled anti-rabbit antibody, and counterstained with propidium iodide. Column 2, propidium iodide staining for cell nuclei. Column 3, cells incubated with affinity-purified MOST-1 polyclonal antibody followed by FITC-labeled anti-rabbit antibody. Column 4, merged images of columns 2 and 3 .

a higher molecular mass isoform of MOST-1 as depicted in Fig. 1A. To confirm this observation, TNT experiments also demonstrated a ladder of different MOST-1 isoforms ranging from 15 to $>100 \mathrm{kDa}$, suggesting the propensity of MOST-1 to form higher molecular mass isoforms (Fig. 1A). In addition, the antibody recognized the largest form of MOST-1 protein. To illustrate that the high molecular mass isoform of MOST-1 was authentic, differential treatment of TNT products was performed. Fig. 1B shows that the high molecular mass isoform of MOST-1 was soluble in SDS, but became insoluble in SDS upon thermal stress. These findings are compatible with the computational analyses which predicted MOST-1 to be unstable as a monomer and poorly soluble.

Localization of MOST-1 to the cytoplasm and the microsomal fraction, cell synchronization, proliferation and apoptosis assays. Fig. 1C demonstrates the presence of MOST-1 protein in both the microsomal and nuclear fractions of the MOLT-4 cell lysate. Immunofluorescence analyses illustrate the cytoplasmic expression of MOST-1 protein (green stain) contrasting with the nuclei (red stain) in four different cell lines (Fig. 2), confirming the cytoplasmic location of MOST-1 protein.

Since we observed MOST-1 expression in cancer cell lines and their normal counterparts, we investigated whether there was a correlation between MOST-1 expression and cell cycle progression. Cell synchronization experiments on normal and malignant breast cell lines synchronized with mimosine demonstrated the apparent cycling of MOST-1 localization with time (Fig. 3 and Table I).

MOST-1 RNAi treatment resulted in significantly decreased MOST-1 transcripts in normal and malignant prostate cell lines as indicated by RT-PCR profiling. Via BrdU labeling, diminished proliferation of DU145 cells was noted following MOST-1 down-regulation. The TUNEL assay revealed a high level of apoptosis in RNAi-treated DU145 cells relative to the other cell lines (data not shown). These observations may be explained by the significant constitutive expression of MOST-1 in the DU145 cell line (1).

The MOST-1 protein interacts with several proteins associated with cancer. Following yeast two-hybrid screening, a total of 
A
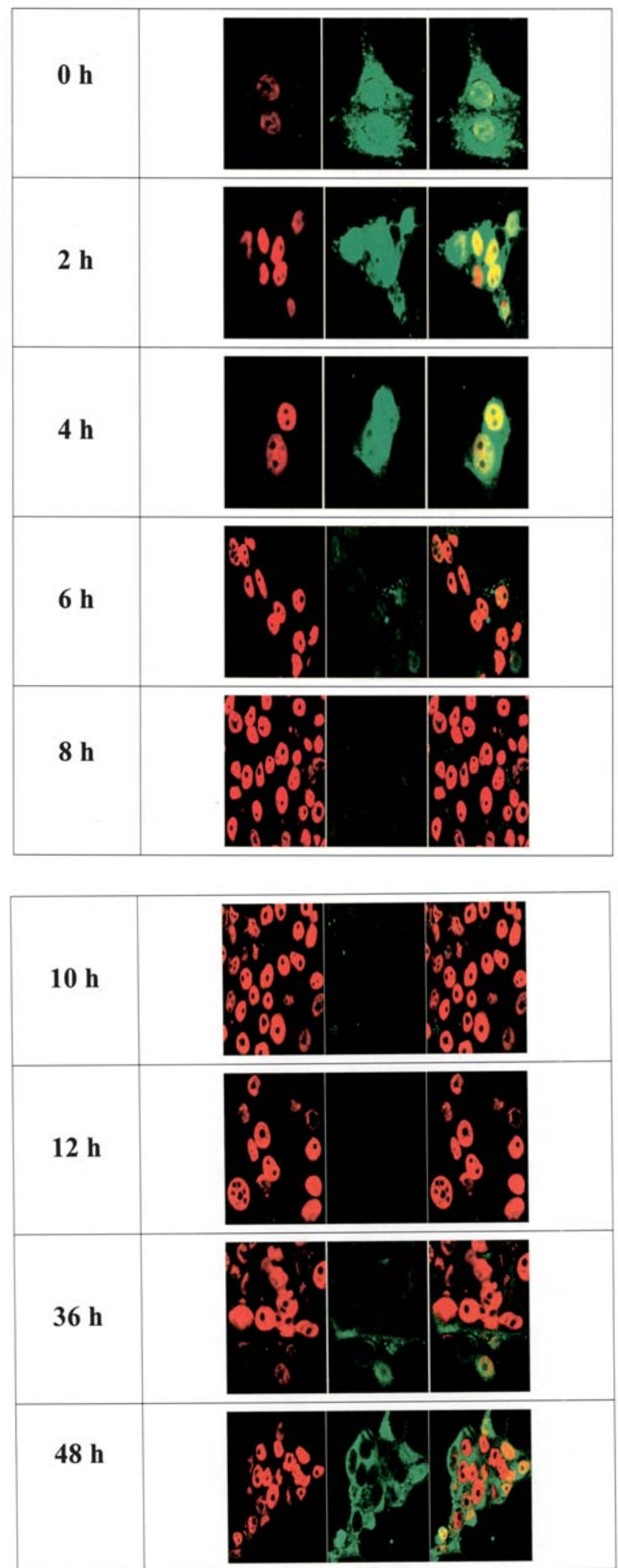

B
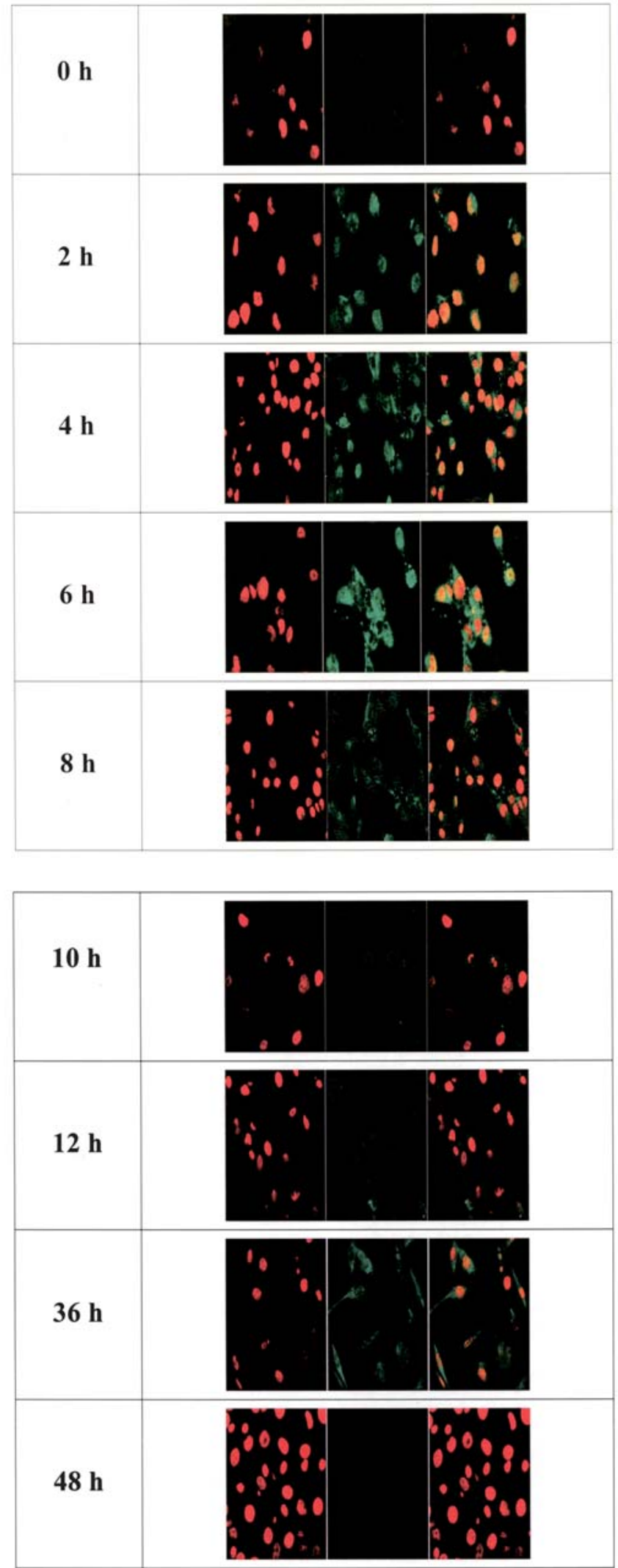

Figure 3. Confocal microscopy following cell cycle synchronization. (A) MCF7 cells observed at 0,2,4, 6, 8, 10, 12, 36 and 48 h after induction of cell cycle by mimosine treatment. (B) Normal mammary cells observed at the same time-points after mimosine treatment. Left, center and right columns as columns $2-4$ in Fig. 2, respectively.

36 MOST-1-interacting clones were obtained, and verified by colony lift assays and PCR amplification (data not shown).
Clones that contained both bait and library plasmids and were positive by colony lift assays were selected and sequenced. 
A

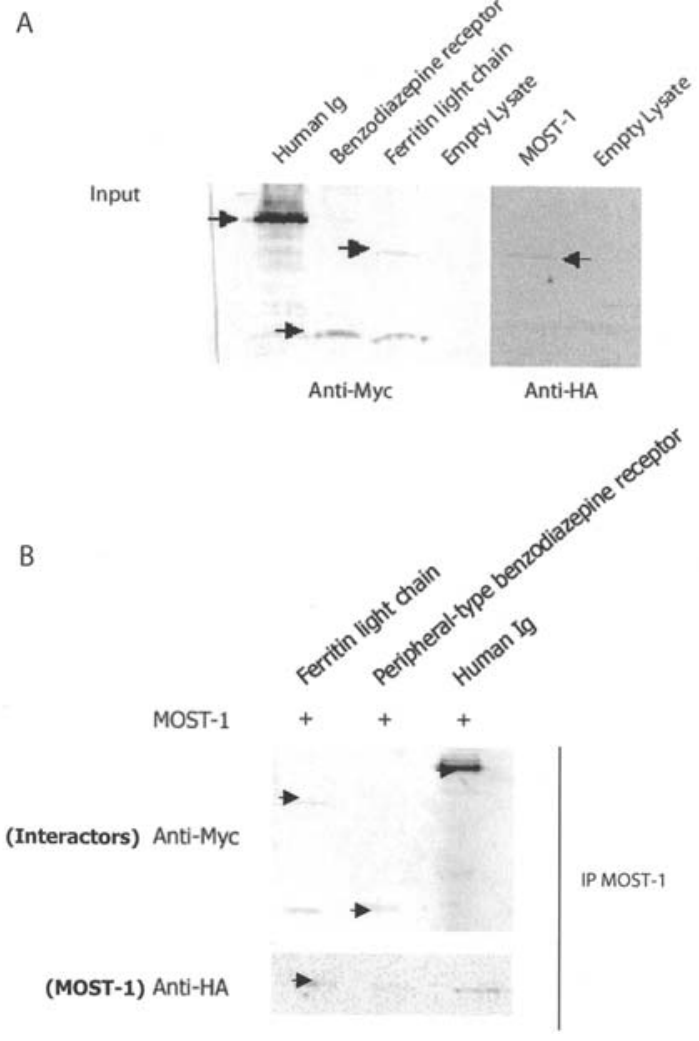

C

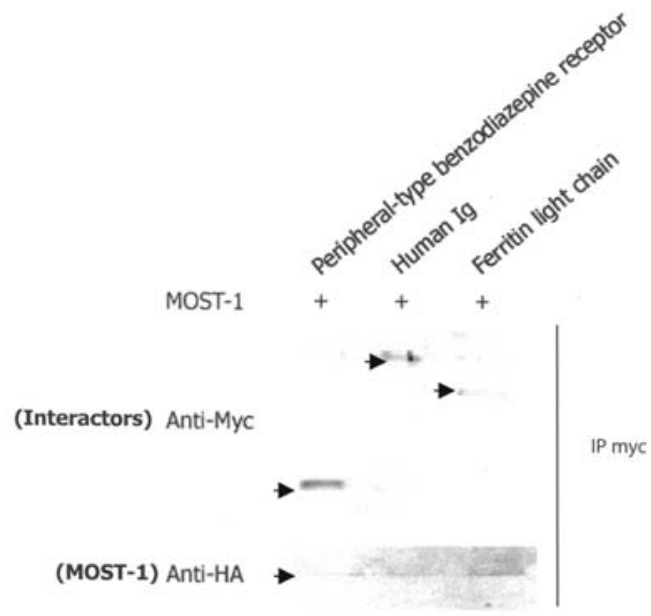

Figure 4. Co-immunoprecipitation of MOST-1 and its interacting proteins. (A) Single expression of individual proteins. Lane 1, cells transfected with pCMVMyc-human-immunoglobulin-C construct. Lane 2, cells transfected with pCMV-Myc-peripheral-type-benzodiazepine receptor construct. Lane 3, cells transfected with pCMV-Myc-ferritin-light-chain construct. Lane 4, untransfected cells. Lanes 1-4, the cell lysates were subjected to SDS-PAGE, the gel transferred onto a membrane and probed with anti-c-Myc tagged with alkaline phosphatase. Lane 5, cells transfected with pCMV-HA-MOST-1 construct. Lane 6, untransfected cells. Lanes 5 and 6, the cell lysates were subjected to SDS-PAGE, the gel transferred onto a membrane, and probed with anti-HA tagged with biotin and detected with streptavidin-alkaline phosphatase. (B) Pull-down experiments using anti-HA antibody. Lane 1, mixed lysates of cells transfected with pCMV-Mycferritin-light-chain and pCMV-HA-MOST-1 constructs. Lane 2, mixed lysates of cells transfected with pCMV-Myc-benzodiazepine-receptor and pCMV-HAMOST-1 constructs. Lane 3, mixed lysates of cells transfected with pCMV-Myc-human-immunoglobulin-C and pCMV-HA-MOST-1 constructs. Lanes 1-3, the cell lysates were incubated with anti-HA monoclonal antibody immobilized onto protein A/G beads. The mixture was washed, subjected to SDS-PAGE, the gel blotted onto a membrane, and incubated with antibodies against Myc and HA tags. (C) Pull-down experiments using anti-Myc antibody. Lane 1, mixed lysates of cells transfected with pCMV-Myc-benzodiazepine-receptor and pCMV-HA-MOST-1 constructs. Lane 2, mixed lysates of cells transfected with pCMV-Myc-human-immunoglobulin-C and pCMV-HA-MOST-1 constructs. Lane 3, mixed lysates of cells transfected with pCMV-Myc-ferritin-light-chain and pCMV-HA-MOST-1 constructs. Lanes 1-3, the cell lysates were incubated with anti-Myc monoclonal antibody immobilized onto protein A/G beads. The mixture was washed, subjected to SDS-PAGE, the gel blotted onto a membrane, and incubated with antibodies against Myc and HA tags.

Of these, overlapping clones were aligned, and seven interactors were identified. The interacting clones comprised creatine kinase (clones overlapped between nt 1192-1555, at the end of the ORF), ferritin light chain (clones overlapped between nt 4-219, encompassing the start of the ORF), peripheral benzodiazepine receptor (nt 156-373), immunoglobulin $\mathrm{C}(\mu)$ and $\mathrm{C}(\delta)$ heavy chain (nt 15191-15331), SNC73 protein (nt 1047-1416), Gardner feline sarcoma v-FGR oncogene (nt 1327-1547), and telethonin (nt 336-555). The authenticity of the MOST-1 interactions was confirmed by co-immunoprecipitation experiments performed with the three strongest interactors based on the number of positive clones and time of appearance. The three strongest interactors, i.e. human immunoglobulin $\mathrm{C}(\mu)$ and $\mathrm{C}(\delta)$ heavy chain, peripheral benzodiazepine receptor and ferritin light chain were cloned into the pCMV-Myc mammalian expression vector, and their expression was verified with anti-Myc antibody (Fig. 4A). Separate pull-down assays using anti-HA and antiMyc antibodies were conducted, and detected with relevant antibodies to confirm the above protein-protein interactions as indicated in Fig. 4B and C.
Table I. Cell synchronization experiments to compare MOST-1 expression level and localization in normal and MCF7 mammary cell lines.

\begin{tabular}{|c|c|c|c|c|}
\hline \multirow[b]{2}{*}{$\begin{array}{l}\text { Time } \\
\text { (h) }\end{array}$} & \multicolumn{2}{|c|}{ Normal mammary cells } & \multicolumn{2}{|c|}{ MCF7 breast cancer cells } \\
\hline & $\begin{array}{c}\text { Expression } \\
\text { level }^{\mathrm{a}}\end{array}$ & Localization $^{\mathrm{b}}$ & $\begin{array}{c}\text { Expression } \\
\text { level }^{\mathrm{a}}\end{array}$ & Localization $^{\mathrm{b}}$ \\
\hline 0 & - & - & +++ & $\mathrm{C}+\mathrm{N}$ \\
\hline 2 & ++ & $\mathrm{C}+\mathrm{N}$ & +++ & $\mathrm{C}+\mathrm{N}$ \\
\hline 4 & ++ & $\mathrm{C}+\mathrm{N}$ & +++ & $\mathrm{C}+\mathrm{N}$ \\
\hline 6 & +++ & $\mathrm{C}+\mathrm{N}$ & ++ & $\mathrm{C}+\mathrm{N}$ \\
\hline 8 & ++ & $\mathrm{C}+\mathrm{N}$ & + & $\mathrm{C}$ \\
\hline 10 & + & $\mathrm{C}$ & + & $\mathrm{C}$ \\
\hline 12 & + & $\mathrm{C}$ & - & - \\
\hline 36 & ++ & $\mathrm{C}+\mathrm{N}$ & + & $\mathrm{C}+\mathrm{N}$ \\
\hline 48 & - & - & ++ & $\mathrm{C}+\mathrm{N}$ \\
\hline
\end{tabular}

${ }^{a}$ Expression level of MOST-1. -, negative; +, faint; ++, moderate; +++ , high. ${ }^{\text {b} C e l l u l a r ~ l o c a l i z a t i o n ~ o f ~ M O S T-1 . ~ C, ~ c y t o p l a s m i c ; ~ N, ~}$ nuclear. 


\section{Discussion}

The cytoplasmic distribution and the microsomal localization of endogenous MOST-1 indicate subcellular compartmentalization of this protein. The in vitro translation experiments demonstrated the propensity of MOST-1 to form high molecular mass isoforms. Based on our data, we propose that the high molecular mass MOST-1 protein consists of self-aggregates. As much as $30 \%$ of all newly synthesized cellular proteins are defective ribosomal products which are degraded by proteosomes shortly after their synthesis in normal or unstressed cells. Thus, there is no accumulation of protein aggregates despite their continued production. The cellular 'quality control' machinery suppresses formation of aggregates by ensuring the fidelity of transcription and translation, the use of chaperones for correct protein folding, and the use of proteosomes for degrading improperly folded polypeptides before they can aggregate (9). However, it is unclear whether aggregation of misfolded proteins is a primary cause or merely a consequence of diseases of protein conformation, although aggregation of misfolded proteins can be a selective process dependent on peptide composition (10). Several proteins which are misfolded or overexpressed have been described, especially in neurodegenerative disorders and many types of cancers. Examples include cystatin $\mathrm{C}$ in susceptible neurons in Alzheimer's disease (11), NuMA which is an important protein for the formation of mitotic centrosomes (12), and CHMP1 which is a nuclear matrix protein affecting chromatin structure and cell cycle progression (13). Overexpression of the NABC1 protein in cancer cells results in detergent-stable homodimers that leads to punctate localization in cultured cells (14). A study involving guanidinobenzoatase shows that aggregation can result in an entity of higher molecular mass accompanied by a change in catalytic activity (15). There are many other examples of native and mutant proteins that undergo aggregation or oligomerization with some physiological or pathological significance (16-22). However, the significance of the tendency of MOST-1 to form high molecular mass isoforms remains to be determined.

Yeast two-hybrid analyses revealed several MOST-1interacting proteins that are either amplified or overexpressed in cancers and are involved in energy metabolism or the cell cycle. One of the stronger interactors with 7 positive clones, creatine kinase isoenzymes, catalyze the synthesis of phosphocreatine by adding a phosphate group to creatine converting it into the high-energy molecule phosphocreatine. The latter is metabolized as a rapid energy source and is subsequently utilized in ATP regeneration in cell types where ATP consumption is rapid or sudden. Creatine kinase is generally found in the cytosol and mitochondria. Different creatine kinase isoforms serve as an energy shuttle between mitochondria and cytoplasmic creatine kinase in discrete cellular sites of high ATP turnover. Furthermore, cellular localization is apparently important for the type of creatine kinase function, suggesting that its interaction with MOST-1 at the appropriate location may have some effect on energy metabolism (23-25). With MOST-1 predominantly found in the membrane-bound fraction, this interaction may be physiologically relevant. Iron is required for normal cell growth and proliferation but excessive iron causes the formation of free radicals. Ferritin acts as the soluble storage form of iron in tissues, with high serum levels occurring in liver disease, infection, inflammation or malignancy (26). Ferritin occurs in discrete pools in the cytosol when not secreted (27). This cellular distribution was also observed for MOST-1, suggesting a potentially functionally relevant interaction. The peripheral benzodiazepine receptor is believed to function in transporting cholesterol across the mitochondrial membrane but current research on its ligands is still in progress to elucidate the physiological relevance of events mediated through such receptors (28). Titin has been reported to control assembly and elasticity in chromosomes. Activated titin kinase phosphorylates telethonin which is a protein of cardiac and skeletal muscle. Telethonin has been identified as a sarcomeric Z-disk protein involved in the reorganization of the cytoskeleton during myofibrillogenesis (29).

MOST-1 interactions with these proteins suggest that MOST-1 may be implicated in energy metabolism. Cell synchronization experiments on normal and malignant breast cell lines synchronized with mimosine displayed MOST-1 localization in the cytoplasm, and suggested a biological 'timing' of MOST-1 expression in view of the complete disappearance of MOST-1 expression at certain time-points, i.e. at 8-12 h for MCF7 cells, at 10-12 h and at $48 \mathrm{~h}$ for normal mammary cells. These findings are compatible with the effects observed in the functional analysis of MOST-1 when its expression is down-regulated. With knockdown of MOST-1 expression in the DU145 prostate cancer cell line, there was decreased cell proliferation but increased cell death implying that the fate of these cancer cells is dependent on MOST-1 levels. Given its potentially efficient translation due to its ideal Kozak sequence (1), altered gene expression and excess MOST-1 protein may enhance the survival of cancer cells. These observations suggest that MOST-1 may act as a mitogen, and may be involved in cancer cell proliferation and progression.

MOST-1 overexpression is associated with high-grade breast and prostate cancers implying its role as a marker for cancer progression (1). A novel prostate-specific gene encoding prostate stem cell antigen (PSCA) which bears homology to a G-protein-coupled receptor is located in $8 \mathrm{q} 24.2$ at the same locus as MOST-1, and is also overexpressed in prostate cancers (30). This suggests the location of prostate cancer-related genes at this locus, which when deregulated, increases the risk of prostate cancer development. Congruent with this notion, our previous cell type profiling showed that MOST-1 is expressed in all human cancer cell lines tested, but is only expressed in specific normal human adult tissues (1). Also noteworthy is the detection of MOST-1 mRNA in human fetal lung but not in adult lung tissue, suggesting that MOST-1 expression is dependent on tissue differentiation (1). A recent report on the correlation between MOST-1 expression and tumor load further supports the involvement of MOST-1 in the process of carcinogenesis. Semiquantitative RT-PCR was employed to study MOST-1 mRNA expression in children with acute lymphoblastic leukemia (ALL). MOST-1 was expressed in the bone marrow mononuclear cells in patients with ALL-L3, and its expression level was related to tumor cell burden suggesting a role for MOST-1 in leukemogenesis of ALL-L3 and as an indicator of tumor burden 
(31). Interestingly, the chimpanzee ortholog of C8orf17 (GenBank accession number DQ035725) was identified to be among positively selected genes in a comparative genomic survey of the genomes of humans and chimpanzees which show only a small overall magnitude of DNA sequence divergence (32). Our study provides a platform for further analysis of MOST-1 functions, the implications of its expression in malignant cells, and its role in the pathway of cancer progression.

\section{Acknowledgments}

We wish to thank B.H. Bay and W.M. Yeo for their assistance. This study was supported by a grant from the National University of Singapore. J.M.M. Tan acknowledges receipt of a research scholarship from the National University of Singapore.

\section{References}

1. Tan JMM, Tock EPC and Chow VTK: The novel human MOST-1 (C8orf17) gene exhibits tissue specific expression, maps to chromosome 8q24.2, and is overexpressed/amplified in high grade cancers of the breast and prostate. Mol Pathol 56: 109-115, 2003.

2. Ethier SP: Identifying and validating causal genetic alterations in human breast cancer. Breast Cancer Res Treat 78: 285-287, 2003.

3. Rao PS, Jaggi M, Smith DJ, Hemstreet GP and Balaji KC: Metallothionein 2A interacts with the kinase domain of PKCmu in prostate cancer. Biochem Biophys Res Commun 310: 1032-1038, 2003.

4. Meehan WJ, Samant RS, Hopper JE, Carrozza MJ, Shevde LA, Workman JL, Eckert KA, Verderame MF and Welch DR: Breast cancer metastasis suppressor 1 (BRMS1) forms complexes with retinoblastoma-binding protein 1 (RBP1) and the $\mathrm{mSin} 3$ histone deacetylase complex and represses transcription. J Biol Chem 279: 1562-1569, 2004.

5. Tai SK, Tan OJ, Chow VT, Jin R, Jones JL, Tan PH, Jayasurya A and Bay BH: Differential expression of metallothionein 1 and 2 isoforms in breast cancer lines with different invasive potential: identification of a novel nonsilent metallothionein-1H mutant variant. Am J Pathol 163: 2009-2019, 2003.

6. Sim DLC and Chow VTK: The novel human HUEL (C4orf1) gene maps to chromosome 4p12-p13 and encodes a nuclear protein containing the nuclear receptor interaction motif. Genomics 59: 224-233, 1999.

7. Quek HH and Chow VTK: Molecular and cellular studies of the human homolog of the $160-\mathrm{kD} \alpha$-subunit of the coatomer protein complex. DNA Cell Biol 16: 275-280, 1997.

8. Sim DLC, Yeo WM and Chow VTK: The novel human HUEL (C4orf1) protein shares homology with the DNA-binding domain of the XPA DNA repair protein and displays nuclear translocation in a cell cycle-dependent manner. Int J Biochem Cell Biol 34: 487-504, 2002.

9. Kopito RR: Aggresomes, inclusion bodies and protein aggregation. Trends Cell Biol 10: 524-530, 2000.

10. Milewski MI, Mickle JE, Forrest JK, Stanton BA and Cutting GR: Aggregation of misfolded proteins can be a selective process dependent upon peptide composition. J Biol Chem 277: 34462-34470, 2002.

11. Deng A, Irizarry MC, Nitsch RM, Growdon JH and Rebeck GW: Elevation of cystatin $\mathrm{C}$ in susceptible neurons in Alzheimer's disease. Am J Pathol 159: 1061-1068, 2001.

12. Gobert GN, Hueser CN, Curran EM, Sun QY, Glinsky VV, Welshons WV, Eisenstark A and Schatten H: Immunolocalization of NuMA and phosphorylated proteins during the cell cycle in human breast and prostate cancer cells as analyzed by immunofluorescence and postembedding immunoelectron microscopy. Histochem Cell Biol 115: 381-395, 2001.

13. Stauffer DR, Howard TL, Nyun T and Hollenberg SM: CHMP1 is a novel nuclear matrix protein affecting chromatin structure and cell-cycle progression. J Cell Sci 114: 2383-2393, 2001.
14. Beardsley DI, Kowbel D, Lataxes TA, Mannino JM, Xin H, Kim WJ, Collins C and Brown KD: Characterization of the novel amplified in breast cancer-1 (NABC1) gene product. Exp Cell Res 290: 402-413, 2003.

15. Poustis-Delpont C, Thaon S, Auberger P, Gerardi-Laffin C, Sudaka P and Rossi B: Monomeric 55-kDa guanidinobenzoatase switches to a serine proteinase activity upon tetramerization. Tetrameric proteinase SP $220 \mathrm{~K}$ appears as the native form. J Biol Chem 269: 14666-14671, 1994.

16. Brown-Augsburger P, Hartshorn K, Chang D, Rust K, Fliszar C, Welgus HG and Crouch EC: Site-directed mutagenesis of Cys15 and Cys-20 of pulmonary surfactant protein D. Expression of a trimeric protein with altered anti-viral properties. J Biol Chem 271: 13724-13730, 1996

17. Lombard N, Swart AC, van der Merwe MJ and Swart P: Sheep adrenal cytochrome b5: active as a monomer or a tetramer in vivo? Endocr Res 28: 485-492, 2002.

18. Trebak M, Begg GE, Chong JM, Kanazireva EV, Herlyn D and Speicher DW: Oligomeric state of the colon carcinoma-associated glycoprotein GA733-2 (Ep-CAM/EGP40) and its role in GA733mediated homotypic cell-cell adhesion. J Biol Chem 276: 2299-2309, 2001

19. Surroca Y, Haverkamp J and Heck AJ: Towards the understanding of molecular mechanisms in the early stages of heat-induced aggregation of beta-lactoglobulin AB. J Chromatogr A 970: 275-285, 2002.

20. Mulero JJ, Yeung G, Nelken ST, Bright JM, McGowan DW and Ford JE: Biochemical characterization of CD39L4. Biochemistry 39: 12924-12928, 2000.

21. Lam LP, Chow RY and Berger SA: A transforming mutation enhances the activity of the c-Kit soluble tyrosine kinase domain. Biochem J 338: 131-138, 1999.

22. Zhao X, Ghaffari S, Lodish H, Malashkevich VN and Kim PS: Structure of the Bcr-Abl oncoprotein oligomerization domain. Nat Struct Biol 9: 117-120, 2002.

23. Shen W, Willis D, Zhang Y, Schlattner U, Wallimann T and Molloy GR: Expression of creatine kinase isoenzyme genes during postnatal development of rat brain cerebellum: evidence for transcriptional regulation. Biochem J 367: 369-380, 2002.

24. De Groof AJ, Fransen JA, Errington RJ, Willems PH, Wieringa B and Koopman WJ: The creatine kinase system is essential for optimal refill of the sarcoplasmic reticulum $\mathrm{Ca}^{2+}$ store in skeletal muscle. J Biol Chem 277: 5275-5284, 2002.

25. Manos P and Bryan GK: Cellular and subcellular compartmentation of creatine kinase in brain. Dev Neurosci 15: 271-279, 1993.

26. Tsuji Y, Ayaki H, Whitman SP, Morrow CS, Torti SV and Torti FM: Coordinate transcriptional and translational regulation of ferritin in response to oxidative stress. Mol Cell Biol 20: 5818-5827, 2002.

27. Parthasarathy N, Torti SV and Torti FM: Ferritin binds to light chain of human H-kininogen and inhibits kallikrein-mediated bradykinin release. Biochem J 365: 279-286, 2002.

28. Chaki S, Funakoshi T, Yoshikawa R, Okuyama S, Okubo T, Nakazato A, Nagamine $M$ and Tomisawa K: Binding characteristics of $\left[{ }^{3} \mathrm{H}\right] \mathrm{DAA} 1106$, a novel and selective ligand for peripheral benzodiazepine receptors. Eur J Pharmacol 371: 197-204, 1999.

29. Mayans O, van der Ven PF, Wilm M, Mues A, Young P, Furst DO, Wilmanns $M$ and Gautel M: Structural basis for activation of the titin kinase domain during myofibrillogenesis. Nature 395: 863-869, 1998

30. Xu LL, Stackhouse BG, Florence K, Zhang W, Shanmugam N, Sesterhenn IA, Zou Z, Srikantan V, Augustus M, Roschke V, Carter K, McLeod DG, Moul JW, Soppett D and Srivastava S: PSGR, a novel prostate-specific gene with homology to a G protein-coupled receptor, is overexpressed in prostate cancer. Cancer Res 60: 6568-6572, 2000.

31. Gao J, Yuan LX, Sun L, Li XX and Liao QK: Expression of MOST-1 mRNA in bone marrow mononuclear cells from patients with acute lymphoblastic leukemia. Sichuan Univ J Med Sci 35: 638-640, 2004.

32. Nielsen R, Bustamante C, Clark AG, Glanowski S, Sackton TB, Hubisz MJ, Fledel-Alon A, Tanenbaum DM, Civello D, White TJ, Sninsky J, Adams MD and Cargill M: A scan for positively selected genes in the genomes of humans and chimpanzees. PLoS Biol 3: e170, 2005. 